\begin{tabular}{|c|l|}
\hline Title & Imaging Ripples on Phononic Crystals Reveals A coustic Band Structure and Bloch Harmonics \\
\hline Author(s) & Profunser, Dieter M.; Wright, Oliver B.; Matsuda, Osamu \\
\hline Citation & $\begin{array}{l}\text { Physical Review Letters, 97, 055502 } \\
\text { https://doi.org/40.1103/PhysRevLett.97.055502 }\end{array}$ \\
\hline Issue Date & 2006-08-04 \\
\hline Doc URL & http://hdl.handle.net/2115/14631 \\
\hline Rights & Copyright @2006 A merican Physical Society \\
\hline Type & article \\
\hline File Information & PRL2006-97-5.pdf \\
\hline
\end{tabular}

Instructions for use 


\title{
Imaging Ripples on Phononic Crystals Reveals Acoustic Band Structure and Bloch Harmonics
}

\author{
Dieter M. Profunser, Oliver B. Wright, ${ }^{*}$ and Osamu Matsuda \\ Department of Applied Physics, Graduate School of Engineering, Hokkaido University, Sapporo, 060-8628, Japan
}

(Received 28 February 2006; published 2 August 2006; corrected 21 August 2006)

\begin{abstract}
Broadband surface phonon wave packets on a phononic crystal made up of a microstructured line pattern are tracked in two dimensions and in real time with an ultrafast optical technique. The eigenmode distribution and the 2D acoustic band structure are obtained from spatiotemporal Fourier transforms of the data up to $1 \mathrm{GHz}$. We find stop bands at the zone boundaries for both leaky-longitudinal and Rayleigh waves, and show how the structure of individual acoustic eigenmodes in $\mathbf{k}$ space depends on Bloch harmonics and on mode coupling.
\end{abstract}

DOI: 10.1103/PhysRevLett.97.055502

The control of sound by propagation in periodic media shows great promise in filtering, shielding, and waveguiding technologies $[1,2]$. Phonons in these phononic crystals experience a spatially modulated elastic impedance, leading to behavior analogous to that of light in photonic crystals or of electrons in solids. The dispersion relation in these periodic systems, whether phononic, photonic, or electronic, depends on their symmetry and geometry, allowing one to tailor desirable characteristics such as band gaps for any propagation direction. Phononic crystals, with three intrinsic acoustic polarizations, promise new means for manipulation of vibrational waves.

Initial interest in phononic crystals was kindled by interdigital transducers, now ubiquitous in cellular phones [3]. Exhibiting 1D periodicity, these microscopic rows of thin metal bars on piezoelectric substrates can resonantly launch and detect surface acoustic waves (SAW's) at GHz frequencies. Stop bands in the frequency spectrum are exploited to fabricate surface wave reflectors and cavities. Similar zone-folded band structure was noted for $\mathrm{THz}$ bulk waves in superlattices, another form of 1D phononic crystal [4]. Progress in optically analogous 2D and 3D photonic crystal technologies [5] spurred developments in phononics: the existence of complete phononic band gaps in arrays of holes, cylinders, or beads was established by acoustic transmission experiments up to the $\mathrm{GHz}$ range [6-9]. By breaking the translational symmetry through the introduction of defects, waveguides, or thin slabs, precise control of sound fields has been achieved [10,11].

The ideal way to measure the sound field in a periodic structure is to image the amplitude and phase of the field over space and time. Steps towards this have been made by optical probing: spatial-amplitude or time-angle scanning elucidates the regions of energy localization and features of the band structure $[12,13]$; these and other optical techniques $[14,15]$ for studying phononic crystals do not, however, achieve the goal of the total spatiotemporal imaging of the wave field. In this Letter we map surface phonons in real time in two dimensions at frequencies up to $1 \mathrm{GHz}$ on a phononic crystal, allowing for the first time direct access to the full 2D band structure and Bloch harmonics.
PACS numbers: 63.20.Dj, 62.65.+k, 63.22.+m, 68.35.Iv

The phononic crystal consists of a grating of alternate $2 \mu \mathrm{m}$-wide copper and silicon oxide lines of thickness $800 \mathrm{~nm}$, both deposited on a silicon (100) substrate (of thickness $0.74 \mathrm{~mm}$ ) perpendicular to the [011] direction [see Fig. 1(a)]. The fabrication, based on a damascene process, involves lithography and chemical-mechanical polishing to a flatness $<10 \mathrm{~nm}$ [16]. A $25 \mathrm{~nm}$ layer of tantalum serves as a diffusion barrier. We sputtered a $30 \mathrm{~nm}$ gold film on top to produce a layer of uniform optical reflectivity. The $\mathrm{Au}$ and Ta layers do not significantly affect the SAW dispersion $[17,18]$. An optical micrograph of the sample before sputtering is shown in Fig. 1(b). The 1D patterning is ideal for identifying the effects of periodicity when imaging in 2D. We use an optical pump and probe technique combined with a common-path interferometer [19]. Excitation (pump) pulses of wavelength $400 \mathrm{~nm}$, energy $\sim 0.2 \mathrm{~nJ}$, duration $200 \mathrm{fs}$, and repetition rate $76.3 \mathrm{MHz}$ are focused at normal incidence to a $\sim 2 \mu \mathrm{m}$ diameter spot on the center of a $\mathrm{Cu}$ line on the sample using a $\times 50$ objective lens [see Fig. 1(c)]. These excite broadband surface acoustic wave packets through the thermoelastic effect at frequencies up to $\sim 1 \mathrm{GHz}$, with a maxi-

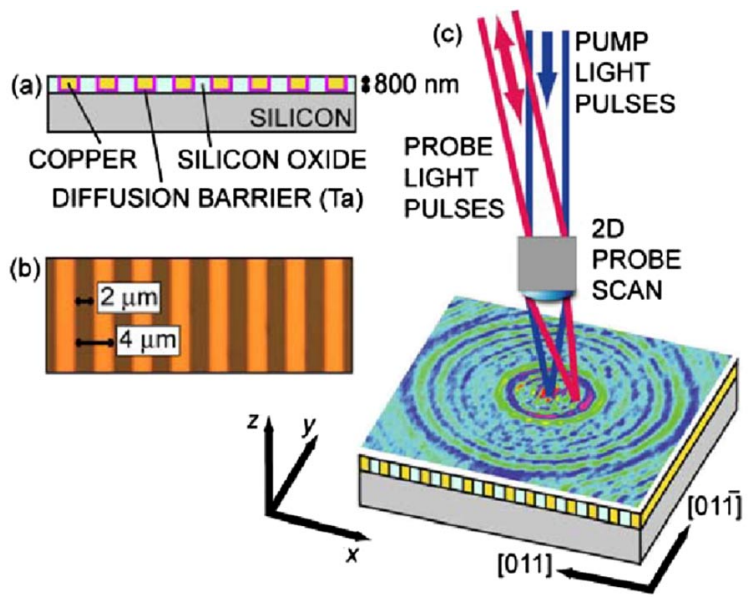

FIG. 1 (color online). (a) Cross section of the specimen. (b) Optical micrograph of the specimen before coating with Au. (c) Schematic experimental arrangement. 
mum amplitude at $\sim 400 \mathrm{MHz}$ (wavelengths $\Lambda \sim 10 \mu \mathrm{m}$ ). These wave packets consist of a coherent superposition of surface-bound eigenmodes of the phononic crystal with an omnidirectional wave vector distribution. Out-of-plane motion is detected with $\sim 0.5 \mathrm{pm}$ resolution by two probe pulses of wavelength $800 \mathrm{~nm}$ at an interval of $\tau=330 \mathrm{ps}$, derived from the same laser as the pump. The $\sim 2 \mu \mathrm{m}$ diameter probe spot is scanned over the sample, and the optical phase difference $\Delta \phi$ between the two probe pulses is imaged at a fixed pump-probe delay time $t$. By incrementing $t$, an animation of the SAW's [20] consisting of 40 frames (each $150 \times 150$ pixels) is obtained within the $13.1 \mathrm{~ns}$ laser repetition period. For the gold top layer and value of $\tau$ used, $\Delta \phi$ is proportional to the time derivative of the out-of-plane surface motion $\left(\partial u_{z} / \partial t\right)$.

A typical image of $\Delta \phi$ over a $150 \mu \mathrm{m} \times 150 \mu \mathrm{m}$ region is shown in Fig. 2(a) for $t=4.6 \mathrm{~ns}$. The multiple wave fronts are caused by the pulse repetition, multimode excitation, and velocity dispersion. An angle-time $(\psi-t)$ representation [13], shown in Fig. 2(b) for a radial distance $r=25 \mu \mathrm{m}$, is convenient for revealing the wave-field symmetry. The structural anisotropy leads to twofold symmetry (discussed later), overriding the residual fourfold symmetry from the Si substrate [21,22]. The pattern is
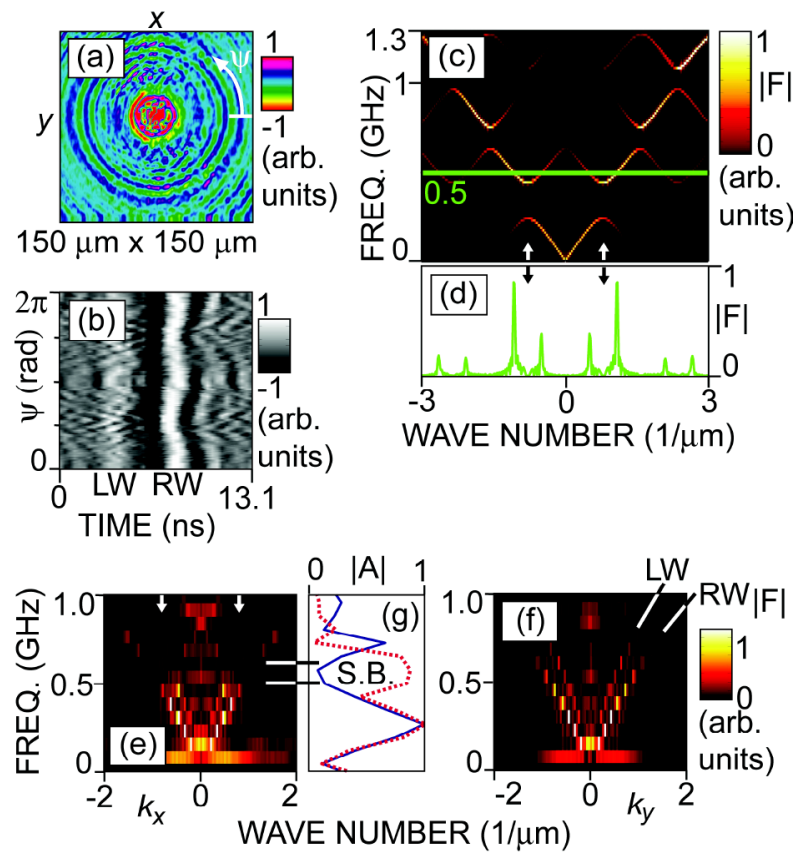

FIG. 2 (color online). Experimental SAW images for the 1D phononic crystal: (a) in $x-y$ form for $t=4.6 \mathrm{~ns}$; (b) in angletime form for $r=25 \mu \mathrm{m}$ (with identified wave fronts). (c) Spatiotemporal Fourier transform $|F|$ of the theoretically calculated displacement field for shear waves in a superlattice with $2 \mu \mathrm{m}$ copper and silicon oxide layers. Arrows: 1st BZ. (d) Cross section of $|F|$ in (c) at $500 \mathrm{MHz}$ horizontal line in (c). (e), (f) Measured $|F|$ for our sample for $x$ - and $y$-directed waves, respectively. (g) Frequency spectra for $x$ - (solid curve) and $y$ (dotted curve) directed waves for $r=20 \mu \mathrm{m}$. S. B.: stop band ( $x$-directed case). complicated by velocity dispersion, showing up particularly strongly in the $\pm y$ directions $(\psi=\pi / 2,3 \pi / 2-$ parallel to the lines) owing to waveguiding effects in the lower-impedance silicon oxide layers [13]. An animation of the $\psi-r$ data gives a graphic visualization of this [20], and allows faster and slower components (discussed later) to be resolved. The higher SAW frequencies (finer ripples) are strongly affected by reflections at the acoustically mismatched interfaces, producing a complex interference pattern of counterpropagating waves [20]; extra attenuation is discernible in the $\pm x$ directions $(\psi=0, \pi)$ through the relative absence of the finer ripples. Overlapping contributions preclude further simple qualitative analysis of the real-time data.

Fourier analysis of the wave field reveals the dispersion relation [23]. Rewriting $\Delta \phi=f(\mathbf{r}, t)$, where $\mathbf{r}$ is the inplane position vector, the $1 \mathrm{D}$ temporal and $2 \mathrm{D}$ spatial Fourier transform $F(\mathbf{k}, \omega)$ is given by

$$
F(\mathbf{k}, \omega)=\frac{1}{(2 \pi)^{3}} \int_{-\infty}^{\infty} \int_{-\infty}^{\infty} f(\mathbf{r}, t) \exp [i(\omega t-\mathbf{k} \cdot \mathbf{r})] d^{2} \mathbf{r} d t
$$

where $\mathbf{k}$ is the 2D wave vector and $\omega$ is the angular frequency. This function is nonzero only for $(\mathbf{k}, \omega)$ satisfying the dispersion relation $\omega= \pm \omega(\mathbf{k})$ for the mode in question [23]. Here we extend this analysis to a 1D phononic crystal, in which case $f(\mathbf{r}, t)$ can be expressed as a Bloch expansion [24]:

$$
\begin{aligned}
f(\mathbf{r}, t)= & \operatorname{Re} \sum_{n} \int_{1 \mathrm{st} \mathrm{BZ}} A_{n}(\mathbf{k}) \sum_{m} C_{n, m}(\mathbf{k}) \\
& \times \exp \left[i\left(\mathbf{k}+\frac{2 \pi m}{p} \mathbf{i}\right) \cdot \mathbf{r}-i \omega_{n}(\mathbf{k}) t\right] d^{2} \mathbf{k},
\end{aligned}
$$

where $m$ is an integer, $p$ is the spatial period, $\mathbf{G}_{0}=2 \pi \mathbf{i} / p$ is a reciprocal lattice vector, and $\mathbf{i}$ is the $x$-directed unit vector. The source functions $A_{n}(\mathbf{k})$, related to the optical excitation and detection, specify the amplitude of a Bloch eigenstate indexed by $\mathbf{k}$ inside the 1st Brillouin zone (BZ) and by branch number $n$. The complex coefficients $C_{n, m}$ determine the amplitude of each Bloch harmonic; $C_{n, m}$ depend on the scattering potential-mainly determined here by the line mechanical properties. To evaluate Eq. (1) with $f(\mathbf{r}, t)$ of Eq. (2), let $C_{n}(\mathbf{k})=C_{n}\left(\mathbf{k}^{\prime}+\right.$ $\left.m \mathbf{G}_{0}\right)=C_{n, m}\left(\mathbf{k}^{\prime}\right)$, for $-\infty<\mathbf{k}<\infty$. The result is

$$
\begin{aligned}
F(\mathbf{k}, \omega)= & \frac{1}{2} \sum_{n}\left[A_{n}\left(\mathbf{k}^{\prime}\right) C_{n}(\mathbf{k}) \delta\left(\omega-\omega_{n}\left(\mathbf{k}^{\prime}\right)\right)\right. \\
& \left.+A_{n}^{*}\left(-\mathbf{k}^{\prime}\right) C_{n}^{*}(-\mathbf{k}) \delta\left(\omega+\omega_{n}\left(\mathbf{k}^{\prime}\right)\right)\right],
\end{aligned}
$$

where $\mathbf{k}^{\prime}=\mathbf{k}-m \mathbf{G}_{0}$ is a vector in the 1 st BZ. This remarkably simple result is the analytical proof that the spatiotemporal Fourier transform of the wave field is proportional to the amplitude of the relevant Bloch harmonic $C_{n}(\mathbf{k})$. Moreover, since $\mathbf{k}$ can be chosen in any $\mathrm{BZ}$, in principle the full range of Bloch harmonics corresponding to $\mathbf{k}-m \mathbf{G}_{0}$ for $\pm m$ can be probed. Each Bloch harmonic 
is weighted by the source function $A_{n}\left(\mathbf{k}^{\prime}\right)$, and the delta function prescribes a finite value for $F$ only when $\omega$ satisfies the extended-zone-scheme dispersion relation.

2D SAW dispersion on 1D phononic crystals can be calculated by numerical simulation [25]. As a simpler example that can be evaluated by semianalytical methods [26], we show in Fig. 2(c) $|F(k, \omega)|$ for nondissipative bulk shear waves propagating (with a sound velocity similar to that of SAW's) normal to a superlattice composed of $2 \mu \mathrm{m}$ copper and silicon oxide layers (as in the present sample). An impulsive, planar acoustic source is assumed. The result resembles a standard dispersion relation in the extended-zone scheme, with evident band gaps, and with maximum $|F|$ at values of $(k, \omega)$ corresponding to an empty-lattice model. However, more information is available: the nonzero amplitudes $|F|$ at any specific $\omega$ on the $|F(k, \omega)|$ plot [see $\omega / 2 \pi=500 \mathrm{MHz}$ in Fig. 2(d)] depend on $C_{n}(k)$ in the Bloch expansion comprised of $k$ separated by a reciprocal lattice vector. This representation of the dispersion relation therefore allows the harmonicity of the periodic potential to be assessed at a glance. It is evident that $C_{n}(k)$ decreases rapidly with $m$ as $k$ deviates from its empty-lattice value by $k \pm m G_{0}$. Although not shown here, one could determine the phase of $F$ to further elucidate the role of the complex $C_{n}(k)$. This example will help in the interpretation of the experiment.

Cross sections of the experimentally derived function $|F|$ for $x$ - and $y$-directed waves are plotted as $f=\omega / 2 \pi$ against $k$ in Figs. 2(e) and 2(f), respectively [27]. The frequency resolution is limited to steps of the repetition rate $76.3 \mathrm{MHz}$. For $x$-directed waves, the periodicity introduces zone boundaries at integral multiples of $k_{x}=$ $\pi / p=0.79 \mu \mathrm{m}^{-1}$, where $p=4 \mu \mathrm{m}$ (see arrows). By comparing Figs. 2(e) and 2(f) one notes a region around $500-600 \mathrm{MHz}$ where the $x$-directed waves are highly attenuated [as we saw in Fig. 2(b)]. These results point to the existence of a stop band. This is confirmed by comparing the curves in Fig. 2(g) representing the modulus of the frequency spectrum (termed $|A|$ ) determined from angletime plots at $r=20 \mu \mathrm{m}$ for angles $\psi=0$ (solid curve$x$ direction) and $90^{\circ}$ (dotted curve $-y$ direction). Two acoustic branches are discernible below $600 \mathrm{MHz}$ in Fig. 2(f). We associate these with Rayleigh-like waves (RW-velocity $\lesssim 4000 \mathrm{~ms}^{-1}$ ) and leaky surface waves of predominantly longitudinal character (LW - velocity $\lesssim$ $\left.7400 \mathrm{~ms}^{-1}\right)$. RW and LW wave fronts are also clearly visible (and identified) in Fig. 2(b). These modes were previously observed by SAW imaging and Brillouin scattering [14,18], and theoretically predicted [28]. Because of the $\mathrm{Si}$, these values are larger than the Rayleigh $\left(2550 \mathrm{~ms}^{-1}\right)$ and bulk longitudinal $\left(5250 \mathrm{~ms}^{-1}\right)$ velocities in the long-wavelength limit for an equivalent polycrystalline $\mathrm{Cu}$ and silicon oxide superlattice [29,30]. (The $\mathrm{Si}$ shifts the band gap to higher $\omega$.) Also noticeable are maxima near $\mathbf{k}=0$ at $\sim 850 \mathrm{MHz}$ in both plots. As explained later, these arise from the Bloch harmonics. Our spectra $A_{n}\left(k^{\prime}\right)$ limit the frequency to a maximum of $\sim 1 \mathrm{GHz}$. (The low- $\omega$ broadband response is due to thermoreflectance.)

The dispersion relation is best perused through the constant- $\omega$ surfaces for $|F(\mathbf{k}, \omega)|$, as shown for four frequencies in Fig. 3. [See [20] for more data.] At $458 \mathrm{MHz}$, when $\Lambda \gtrsim p$, the RW and LW modes form concentric rings, indicating an approximately isotropic $\omega_{n}(\mathbf{k})$. Mode identification is provided on the right in Fig. 3. The RW ring is also visible shifted in the $\pm k_{x}$ directions by $\pm \mathbf{G}_{0}$ owing to the effect of the Bloch harmonics (see dotted lines). These "ghosts" arise through the finite value of $C_{n}(\mathbf{k})$ in Eq. (3) for $\mathbf{k}$ in the 2 nd BZ. $C_{n}(\mathbf{k})$ depend on the grating's scattering potential, which is frequency dependent because of the variable acoustic penetration $(\sim \Lambda)$.

At $534 \mathrm{MHz}$ there is strong evidence for stop bands in both RW and LW propagation for angles $\theta$ (to the $x$ direction) up to $15^{\circ}$ and $20^{\circ}$, respectively. The remaining parts of the rings become noticeably flattened - consistent with the twofold symmetry noted earlier-through the

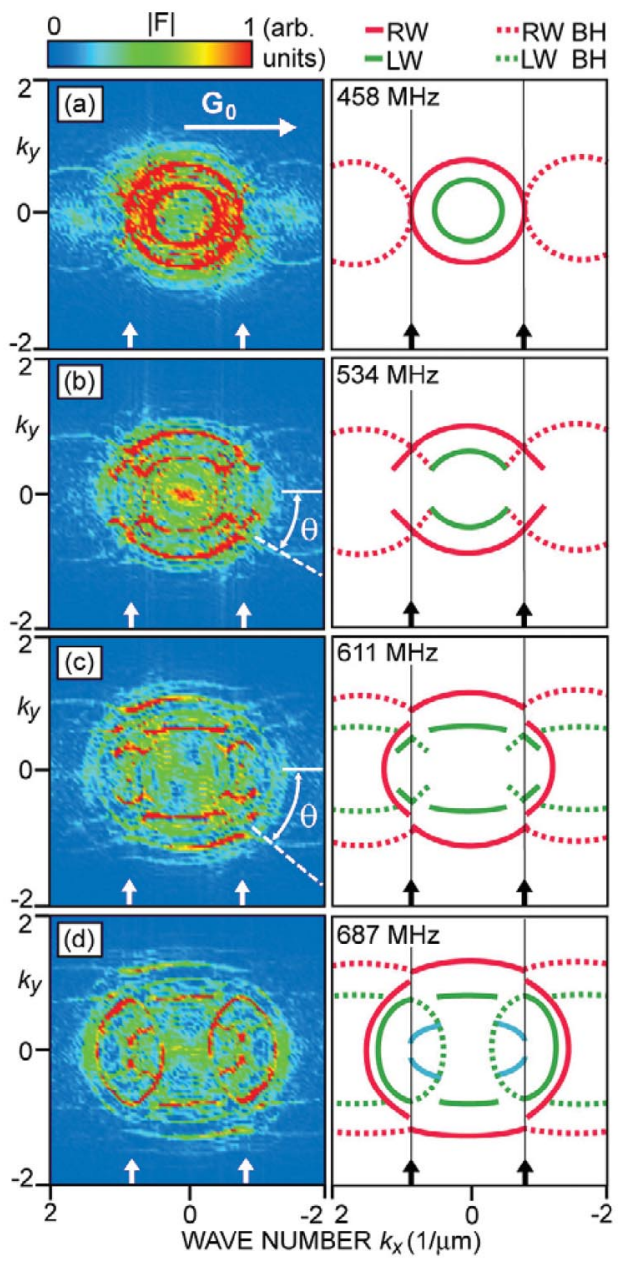

FIG. 3 (color). Experimental constant- $\omega$ surfaces $\left(k_{y}-k_{x}\right)$ for (a) $458 \mathrm{MHz}$, (b) $534 \mathrm{MHz}$, (c) $611 \mathrm{MHz}$, and (d) $687 \mathrm{MHz}$. The upward arrows indicate the 1st BZ. The white dashed lines in (b) and (c) correspond to $\theta=30^{\circ}$ and $40^{\circ}$, respectively. Mode identification is provided on the right. Dotted lines: branches arising from Bloch harmonics (BH). Blue lines: higher-order LW. 
effect of the periodicity (that imposes zero group velocity at the BZ edges), as previously observed for similar 1D phononic structures $[14,15]$. We interpret the features joining the RW and LW branches at $\theta \approx 30^{\circ}$ [white dashed line in Fig. 3(b)] as RW Bloch harmonics inside the 1st BZ. In addition, a peak is observed at $\mathbf{k}=\mathbf{0}$, the origin of which is not yet clear.

At $611 \mathrm{MHz}$ the LW gap persists up to $\theta \approx 20^{\circ}$, whereas RW branches in the 2nd BZ become faintly visible. LW Bloch harmonics also begin to be discernible. In addition, striking discontinuities in both the LW and RW constant- $\omega$ surfaces appear at $\theta \approx 40^{\circ}$ [white dashed line in Fig. 3(c)]. We interpret this as evidence for strong mixing between these modes at this angle; RW and LW are known to hybridize in corrugated gratings due to their common inplane longitudinal stress component [14,15,31]. At $687 \mathrm{MHz}$ the LW gap also closes, and, together with the LW Bloch harmonics inside the 1st BZ, two vertically elongated elliptical shapes are formed. In addition, for $\theta>$ $7^{\circ}$, modes appear inside these ellipses with an intriguingly high phase velocity [up to $\sim 8000 \mathrm{~ms}^{-1}$ - blue lines in Fig. 3(d)] that decreases with increasing frequency [20]. This velocity near that of bulk longitudinal waves in $\mathrm{Si}$ $\left(\sim 9000 \mathrm{~ms}^{-1}\right)$, the turn-on at finite $\omega$, and the velocity dispersion are evidence for these being higher-order LW (associated with the finite grating thickness) [17,28]. To our knowledge these fascinating LW direction dependences, reminiscent of longitudinal modes in superlattices [32], have not been studied in 1D surface phononic crystals such as those consisting of arrays of etched grooves [14,15] or evaporated stripes [3]. At $840 \mathrm{MHz}$ we observe a peak at $\mathbf{k}=\mathbf{0}$ in the constant- $\omega$ surface [20] due to the overlap of the LW Bloch harmonics there.

This direct experimental resolution of the full dispersion relation and Bloch harmonics of a surface phononic crystal raises tantalizing questions as to the physics behind the direction-dependent mode coupling and Bloch modes in such noncorrugated structures. A quantitative analysis would require numerical computation beyond the scope of this Letter. This poses a challenge to theorists and provides a strong incentive for similar studies on other $1 \mathrm{D}$ and 2D phononic crystals, with interesting parallels in other fields such as water waves [33] or photonics [34]. Moreover, our approach opens the way to the direct probing of a new generation of phononic crystal devices. For example, it can be used to measure the acoustic dispersion of guided or defect modes in phononic crystals, or to elucidate the physics of coupled phononic-photonic crystal systems.

We thank Alex Maznev for donating the specimen, and Shin-ichiro Tamura, Madeleine Msall, and Yoshihiro Sugawara for valuable discussions.

*Electronic address: assp@kino-ap.eng.hokudai.ac.jp [1] M. S. Kushwaha, Int. J. Mod. Phys. B 10, 977 (1996).
[2] M. Sigalas, M.S. Kushwaha, and E.N. Economou, Z. Kristallogr. 220, 765 (2005).

[3] F.S. Hickernell, in Physical Acoustics, edited by R.N. Thurston, A.D. Pierce, and E. P. Papadakis (Academic, New York, 1999), Vol. 24, p. 135.

[4] V. Narayanamurti et al., Phys. Rev. Lett. 43, 2012 (1979).

[5] J. D. Joannopoulos, P. R. Villeneuve, and F. Shanhui, Nature (London) 386, 143 (1997).

[6] F. Meseguer et al., Phys. Rev. B 59, 12169 (1999).

[7] D. García-Pablos et al., Phys. Rev. Lett. 84, 4349 (2000).

[8] J. O. Vasseur et al., Phys. Rev. E 65, 056608 (2002).

[9] X. Zhang et al., Appl. Phys. Lett. 88, 041911 (2006).

[10] A. Khelif et al., Phys. Rev. B 65, 174308 (2002).

[11] S. Yang et al., Phys. Rev. Lett. 93, 024301 (2004).

[12] D. C. Hurley, S. Tamura, J. P. Wolfe, and H. Morkoc, Phys. Rev. Lett. 58, 2446 (1987).

[13] R. E. Vines, J. P. Wolfe, and A. V. Every, Phys. Rev. B 60, 11871 (1999).

[14] J. R. Dutcher et al., Phys. Rev. Lett. 68, 2464 (1992).

[15] L. Dhar and J.A. Rogers, Appl. Phys. Lett. 77, 1402 (2000).

[16] The sample was fabricated by SEMATECH Corp.

[17] G.W. Farnell and E. L. Adler, in Physical Acoustics, edited by W.P. Mason and R. N. Thurston (Academic, New York, 1972), Vol. 9, p. 35.

[18] Y. Sugawara et al., Phys. Rev. Lett. 88, 185504 (2002).

[19] T. Tachizaki et al., Rev. Sci. Instrum. 77, 043713 (2006).

[20] See EPAPS Document No. E-PRLTAO-97-025631 or http://kino-ap.eng.hokudai.ac.jp for the animations in $x-$ $y, \psi-r$, and Fourier spaces. For more information on EPAPS, see http://www.aip.org/pubservs/epaps.html.

[21] R. E. Vines, M. R. Hauser, and J. P. Wolfe, Z. Phys. B 98, 255 (1995).

[22] Owing to the Si substrate orientation [see Fig. 1(c)], the Bloch modes arising from Rayleigh-like waves should have pseudo-SAW character near the $x$ and $y$ directions. In these directions the phonon polarization is restricted by symmetry to the sagittal plane. Near the $45^{\circ}$ directions, the Rayleigh-like modes have SAW character, and all modes include tangential polarization components.

[23] Y. Sugawara, O. B. Wright, and O. Matsuda, Appl. Phys. Lett. 83, 1340 (2003).

[24] See, e.g., C. Kittel, Introduction to Solid State Physics (Wiley, New York, 2005), 8th ed.

[25] T. Aono, Y. Tanaka, and S. Tamura, Physica (Amsterdam) B263, 98 (1999).

[26] S. Tamura, D. C. Hurley, and J. P. Wolfe, Phys. Rev. B 38, 1427 (1988).

[27] The data were processed with a $-t$ component as in Ref. [23].

[28] N.E. Glass and A. A. Maradudin, J. Appl. Phys. 54, 796 (1983).

[29] O.L. Anderson, in Physical Acoustics, edited by W.P. Mason (Academic, New York, 1965), Vol. 3B, p. 43.

[30] G. Carlotti, L. Doucet, and M. Dupeux, Thin Solid Films 296, 102 (1997).

[31] L. Giovannini, F. Nizzoli, and A. M. Marvin, Phys. Rev. Lett. 69, 1572 (1992).

[32] Y. Tanaka, M. Narita, and S. Tamura, J. Phys. Condens. Matter 10, 8787 (1998).

[33] X. Hu and C. T. Chan, Phys. Rev. Lett. 95, 154501 (2005).

[34] H. Gersen et al., Phys. Rev. Lett. 94, 123901 (2005). 